\title{
Resultados da aplicabilidade de um protocolo de rastreamento populacional de câncer de próstata em Chapecó-SC
}

\author{
Results of the applicability of a population screening protocol for prostate cancer in Chapecó-SC \\ Resultados de la aplicabilidad de un protocolo de cribado poblacional de cáncer de próstata en
} Chapecó-SC

Recebido: 07/12/2021 | Revisado: 11/12/2021 | Aceito: 14/12/2021 | Publicado: 21/12/2021

\author{
Gustavo Henrique Andrade \\ ORCID: https://orcid.org/0000-0001-9620-294X \\ Universidade Comunitária da Região de Chapecó, Brasil \\ E-mail: gustavo.andrade@unochapeco.edu.br \\ Luiza Pagnussatt \\ ORCID: https://orcid.org/0000-0002-9141-7375 \\ Universidade Comunitária da Região de Chapecó, Brasil \\ E-mail: luiza.p@unochapeco.edu.br \\ Thalis Matheus Fabiani \\ ORCID: https://orcid.org/0000-0002-3602-0017 \\ Universidade Comunitária da Região de Chapecó, Brasil \\ E-mail: thalis.fabiani@unochapeco.edu.br \\ Marcelo Zeni \\ ORCID: https://orcid.org/0000-0002-3072-2106 \\ Universidade Comunitária da Região de Chapecó, Brasil \\ E-mail: urologiachapeco@gmail.edu.br \\ Junir Antonio Lutinski \\ ORCID: https://orcid.org/0000-0003-0149-5415 \\ Universidade Comunitária da Região de Chapecó, Brasil \\ E-mail: junir@unochapeco.edu.br
}

\begin{abstract}
Resumo
Campanhas que estimulam o rastreamento do câncer de próstata no Brasil são realizadas anualmente e preconizam o uso do toque retal associado à dosagem sérica do antígeno prostático específico. Objetivo: Avaliar os resultados e a aplicabilidade de um protocolo de rastreamento do câncer de próstata em Chapecó-SC. Métodos: Foram analisados dados clínicos de questionários de perfil epidemiológico e testes de dosagem de PSA de 249 homens, acima de 45 anos, residentes de Chapecó-SC e conveniados à Unimed, por meio de consulta ao banco de dados e exames do laboratório do Hospital Unimed Chapecó. Resultados: A amostra foi composta por 249 homens cuja média de idade foi de 51,2 anos. A média de valor do PSA da amostra foi de $0,82 \mathrm{ng} / \mathrm{dL}$ e 3,2\% dos pacientes tiveram seu resultado acima de 2,5 ng/dL. 14,05\% possuem histórico de parente de primeiro grau com câncer de próstata. Discussão: Evidências sugerem que não é necessário realizar o rastreio para câncer de próstata. Todavia, nos Estados Unidos, a US Preventive Services Task Force recomendou a não realização do rastreio e houve um aumento de metástases à distância do câncer de próstata em indivíduos com idade superior a 75 anos. A recomendação mais atual indica que deve ser feito de forma individualizada em pacientes entre 55 e 69 anos, para diagnóstico em estágio inicial e redução do sobrediagnóstico e overtreatment. Conclusão: Diante dos dados apresentados, a individualização no rastreamento é mais importante que o rastreamento populacional indiscriminado.
\end{abstract}

Palavras-chave: Neoplasia de próstata; Programas de rastreamento; Antígeno Prostático Específico.

\begin{abstract}
Campaigns that encourage screening for prostate cancer in Brazil are carried out annually and advocate the use of digital rectal examination associated with serum dosage of prostate-specific antigen. Objective: To evaluate the results and applicability of a prostate cancer screening protocol in Chapecó-SC. Methods: Clinical data from epidemiological profile questionnaires and PSA dosage tests of 249 men, over 45 years of age, residents of Chapecó-SC and affiliated to Unimed, were analyzed by consulting the Hospital's database and laboratory tests Unimed Chapecó. Results: The sample consisted of 249 men whose mean age was 51.2 years. The mean PSA value of the sample was $0.82 \mathrm{ng} / \mathrm{dL}$ and $3.2 \%$ of the patients had their result above $2.5 \mathrm{ng} / \mathrm{dL}$. $14.05 \%$ have a history of a first-degree relative with prostate cancer. Discussion: Evidence suggests that screening for prostate cancer is not necessary. However, in the United States, the US Preventive Services Task Force recommended not performing screening and there was an increase in distant metastases of prostate cancer in individuals over 75 years of age. The most current recommendation indicates that it
\end{abstract}


should be done individually in patients between 55 and 69 years old, for early-stage diagnosis and reduction of overdiagnosis and overtreatment. Conclusion: In view of the data presented, individualization in tracking is more important than indiscriminate population tracking.

Keywords: Prostate cancer; Screening programs; Prostate-Specific Antigen.

\section{Resumen}

Anualmente se llevan a cabo campañas que fomentan la detección del cáncer de próstata en Brasil y abogan por el uso del tacto rectal asociado a la dosis sérica de antígeno prostático específico. Objetivo: Evaluar los resultados y la aplicabilidad de un protocolo de cribado de cáncer de próstata en Chapecó-SC. Métodos: Los datos clínicos de los cuestionarios de perfil epidemiológico y las pruebas de dosificación de PSA de 249 hombres, mayores de 45 años, residentes de Chapecó-SC y afiliados a Unimed, fueron analizados mediante la consulta de la base de datos del Hospital y las pruebas de laboratorio Unimed Chapecó. Resultados: La muestra estuvo formada por 249 hombres cuya edad media fue de 51,2 años. El valor medio de PSA de la muestra fue de 0,82 ng / dL y el 3,2\% de los pacientes tuvo su resultado por encima de 2,5 ng / dL. El 14,05\% tiene antecedentes de un familiar de primer grado con cáncer de próstata. Discusión: La evidencia sugiere que la detección del cáncer de próstata no es necesaria. Sin embargo, en los Estados Unidos, el Grupo de Trabajo de Servicios Preventivos de EE. UU. Recomendó no realizar exámenes de detección y hubo un aumento en las metástasis a distancia del cáncer de próstata en personas mayores de 75 años. La recomendación más actual indica que debe realizarse de forma individual en pacientes entre 55 y 69 años, para el diagnóstico precoz y reducción del sobrediagnóstico y sobretratamiento. Conclusión: en vista de los datos presentados, la individualización en el seguimiento es más importante que el seguimiento indiscriminado de la población.

Palabras clave: Neoplasia de próstata; Programas de seguimiento; Antígeno específico de la próstata.

\section{Introdução}

No Brasil, campanhas que incentivam o rastreamento do câncer de próstata, com a dosagem do antígeno prostático específico (PSA - sigla em inglês) e exame digital retal, têm sido realizadas anualmente, a partir dos 50 anos, conhecidas como "Novembro Azul” como descrito pela Sociedade Brasileira de Urologia (2017), cujo objetivo é realizar o diagnóstico precoce, aumentando, teoricamente, a probabilidade do sucesso do tratamento, a sobrevida e a qualidade de vida do paciente, como descrito por Steffen (2018). É importante destacar que, quanto maior o tempo de seguimento, há maiores benefícios, sendo um único rastreio insuficiente para ter efeito na mortalidade, como observado por Hugosson (2019).

Entretanto, em autópsias, cerca de metade dos homens acima de 80 anos possuíam células malignas na próstata, apesar de não apresentarem nenhum sintoma em vida, como destacado por Grossman (2018). Soma-se a isso a metanálise publicada pela biblioteca Cochrane em 2013 que identificou que a dosagem do PSA em pacientes assintomáticos não diminui, de forma significativa, a mortalidade a longo prazo, como descrito por Ilic (2013).

Dessa forma, o objetivo dessa pesquisa é avaliar os resultados de um protocolo de rastreamento de câncer de próstata em curto prazo, correlacionando os dados coletados com a sua aplicabilidade.

\section{Metodologia}

Trata-se de um estudo quantitativo, observacional, descritivo, retrospectivo e transversal. A coleta de dados foi realizada por meio de consulta e análise ao banco de dados gerados pelo projeto de detecção precoce do câncer de próstata realizado no período de 01 de dezembro de 2018 a 31 de março de 2019 pela Unimed Chapecó. Foram considerados elegíveis, para entrada no projeto, homens, acima de 45 anos, beneficiários da Unimed e residentes no município de Chapecó. Foram incluídos os pacientes que completaram as etapas do projeto e que aceitaram participar da coleta de dados, assinando o Termo de Consentimento Livre e Esclarecido (TCLE). Foram excluídos aqueles que não foram localizados pelo projeto de detecção precoce de câncer de próstata da Unimed Chapecó ou não completaram todas as etapas do projeto.

Estudos quantitativos são mais indicados para o planejamento de ações coletivas, pois seus resultados são passíveis de generalização, principalmente quando as amostras pesquisadas representam, com fidelidade, a população de onde foram retiradas . No estudo observacional o investigador atua meramente como expectador de fenômenos ou fatos, sem, no entanto, realizar 
qualquer intervenção que possa interferir no curso natural e/ou no desfecho dos mesmos (Fontelles et al., 2009).

O protocolo consistiu em dosagem do PSA e coleta de dados individuais de perfil epidemiológico, como idade, etnia, índice de massa corporal (IMC), presença de doenças pré-existentes, histórico de câncer de próstata em parentes de primeiro grau, histórico de procedimentos cirúrgicos, presença de sintomas urinários, uso contínuo de medicamentos alfa-bloqueadores ou inibidores da 5-alfa-redutase. Indivíduos que tinham dosagem de PSA alterada (PSA total > 2,5 ng/dL) e/ou aqueles que não tinham, porém com fatores de risco identificados, foram avaliados em consultas individuais com médicos urologistas cooperados da Unimed Chapecó. Homens com dosagem de PSA considerada normal ou que não possuíam fatores de risco receberam apenas orientações.

A análise estatística foi realizada utilizando o programa PAST, versão 4.02, e apresentada sob a forma de gráficos e tabelas através do teste de Spearman, teste T e Shapiro-Wilk. As variáveis qualitativas foram ilustradas em frequências absolutas (n) e relativas (percentual). O intervalo de confiança adotado foi de $95 \%$, poder de estudo de $80 \%$ e significância estatística p $\leq$ 0,05 .

O projeto foi desenvolvido de acordo com a Resolução CNS 466/12 Resolução 466, de 12 de dezembro de 2012 e submetido para o Comitê de Ética em Pesquisa envolvendo Seres Humanos da Universidade Comunitária da Região de Chapecó e para o comitê de ética do Hospital Unimed Chapecó. A coleta e análise de dados somente ocorreu após aprovação do projeto pelo comitê, cujo número do protocolo de aprovação é 3.691.824.

\section{Resultados e Discussão}

No período estudado, 500 pacientes foram arrolados e considerados elegíveis pelos critérios previstos. Destes, 251 pacientes foram excluídos, por não cumprirem todos os critérios de inclusão, resultando em uma amostra de 249 homens que aceitaram participar do projeto de deteç̧ão precoce de câncer de próstata realizado pelo Hospital Unimed Chapecó, entre 01 de dezembro de 2018 e 31 de março de 2019.

Em relação aos valores de PSA coletados da amostra, 219 (88\%) tiveram o resultado até 1,5 ng/dL, $22(8,8 \%)$ de 1,5 até $2,5 \mathrm{ng} / \mathrm{dL}$ e $8(3,2 \%)$ tiveram resultado acima de $2,5 \mathrm{ng} / \mathrm{dL}$. A média encontrada foi de $0,82 \mathrm{ng} / \mathrm{dL}$, sendo $0,12 \mathrm{ng} / \mathrm{dL}$ o menor e $6,55 \mathrm{ng} / \mathrm{dL}$ o maior valor encontrado.

A faixa etária dos pacientes variou entre 46 e 84 anos, demonstrada na figura 1, sendo a média de 51,2 anos. De acordo com o Teste de Spearman, houve significância estatística, com p = 0,007, ocorrendo aumento do PSA conforme maior faixa etária. 
Figura 1 - Distribuição dos indivíduos da amostra conforme faixa etária.

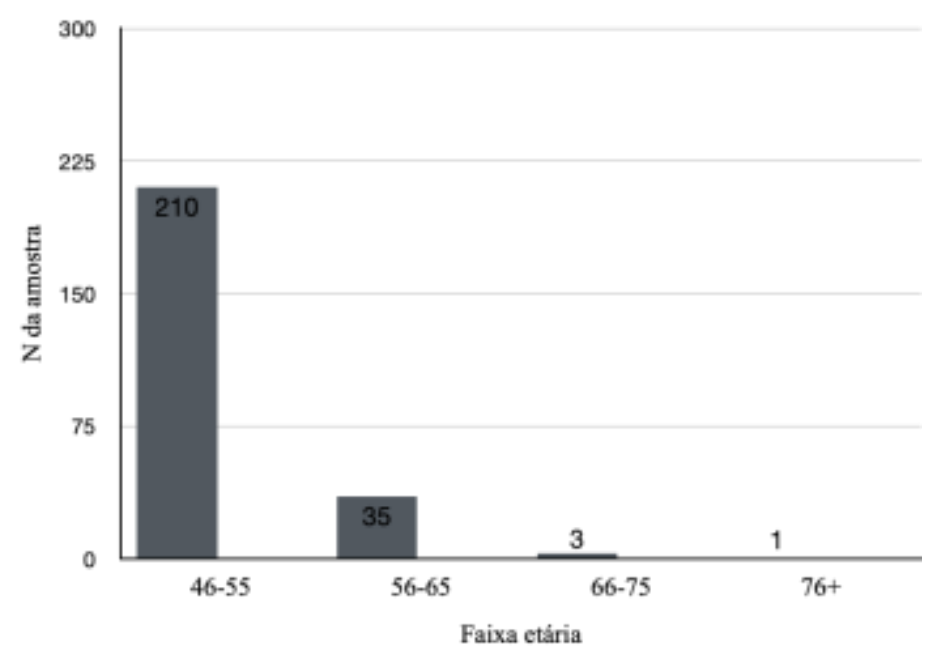

Fonte: Autores (2020).

Em relação à etnia, $184(73,9 \%)$ pacientes se declararam brancos, $35(14,1 \%)$ se declararam pardos, $24(9,6 \%)$ se declararam negros, $3(1,2 \%)$ se declararam amarelos e $3(1,2 \%)$ não responderam. A análise estatística não mostrou diferença entre brancos, pardos, negros e amarelos.

Em relação a comorbidades, 194 (77,9\%) pacientes não responderam. Dentre os que responderam, 33 (13,25\%) hipertensão arterial sistêmica, $13(5,22 \%)$ possuem diabetes mellitus tipo 2, $13(5,22 \%)$ são tabagistas, 5 (2\%) obesidade e 4 $(1,6 \%)$ apresentam cardiopatias.

Na amostra coletada, $5(2,1 \%)$ pacientes tiveram histórico prévio positivo para outros tipos de cânceres, 204 (81,9\%) tiveram histórico prévio negativo e 40 não responderam (16\%). Em relação ao uso de medicamentos, 63 (25,3\%) pacientes usam medicação contínua, 184 (73,8\%) não usam e 2 (0,9\%) não responderam. Quanto ao histórico prévio de procedimentos cirúrgicos, 107 (42,9\%) já realizaram, sendo somente 1 (0,93\%) na próstata, 137 (55\%) não realizaram e 5 (2,1\%) não responderam.

No quesito histórico de câncer de próstata em parentes de primeiro grau, 35 (14,05\%) pacientes responderam que sim, $210(84,3 \%)$ não e $4(1,65 \%)$ não responderam, no entanto, p = 0,81, não havendo significância do valor do PSA e histórico em parentes de primeiro grau.

Na Tabela 2, demonstra-se a sintomatologia referida pela amostra.

Tabela 2 - Porcentagem referente à presença de cada sintomatologia nos pacientes do Hospital Unimed Chapecó que participaram do projeto de detecção precoce de câncer de próstata.

\begin{tabular}{llll}
\hline Variável & Sim & Não & Não responderam \\
\hline Dor ao urinar & $4,01 \%$ & $92,77 \%$ & $3,21 \%$ \\
Necessidade frequente ou urgente de urinar & $5,22 \%$ & $93,97 \%$ & $0,8 \%$ \\
Aumento da frequência de urinar à noite & $4,81 \%$ & $93,57 \%$ & $1,6 \%$ \\
Necessidade de esforço para urinar & $4,01 \%$ & $95,18 \%$ & $0,8 \%$ \\
Não consegue esvaziar completamente a bexiga & $7,62 \%$ & $90,76 \%$ & $1,6 \%$ \\
Há presença de sangue na urina & $0 \%$ & 99,19 & $0,81 \%$ \\
Dor na região dos testículos ou perto do ânus & $2,81 \%$ & $96,30 \%$ & $0,8 \%$ \\
\hline
\end{tabular}

Fonte: Autores (2020). 
Nenhum dos pacientes da amostra, após avaliação clínica e do PSA, foi submetido à biópsia de próstata. Portanto, no grupo de pacientes avaliados, o protocolo de rastreamento não resultou em nenhum diagnóstico de câncer de próstata.

O câncer de próstata é o segundo tipo de câncer mais comum entre os homens no Brasil, ficando atrás apenas do câncer de pele não-melanoma. Sua prevalência é demonstrada pelas estatísticas do Instituto Nacional de Câncer, com 68.220 novos casos em 2018. Além disso, há um aumento na taxa de incidência, o que pode ser justificado, em partes, pela evolução dos métodos diagnósticos, aumento da expectativa de vida e maior propagação de informações à população como descrito pelo INCA - Instituto Nacional de Câncer (2018).

O protocolo de rastreamento de câncer de próstata consiste em dosar o PSA e realizar o exame de toque retal, tanto em indivíduos sintomáticos quanto em assintomáticos, com o propósito de diagnosticar precocemente e como método de diagnóstico em pacientes com sintomas clínicos, tendo alto valor benéfico nesse último caso como visto por Steffen (2018)', sendo que sua realização é defendida há mais de 10 anos no Brasil como observado por Modesto (2017). O toque retal é um exame rápido, realizado em consultório médico, não podendo ser substituído. Ele pode detectar o câncer de próstata com mais eficácia do que propriamente o exame de PSA, como apontado por Sarris (2018).

O PSA é produzido pelas células epiteliais da próstata como descrito por Toebe (2020). É um exame de baixa sensibilidade e especificidade, uma vez que o limiar geralmente usado (>4 ng/mL) apresenta 70\% de resultados falso-positivos como visto por Mulhem (2015) e que 75\% das biópsias de próstata realizadas em pacientes com resultado nessa faixa dão negativo para neoplasia como descrito por Castro (2011). Ademais, ele é um marcador órgão-específico e não doença-específico, dessa forma, a prostatite e hiperplasia prostática também podem elevar seus níveis séricos como visto por Silva (2017).

Utilizam-se valores acima de $4 \mathrm{ng} / \mathrm{ml}$ para direcionar a indicação de biópsia, embora não exista um consenso sobre o limiar de PSA a ser considerado, visto que alguns estudos demonstraram maior sensibilidade utilizando o limiar de 2,5 ng/ml, como descrito por Demuner (2021). A média obtida dos valores de PSA na amostra foi de 0,82, estando dentro da normalidade. Do total, somente 2 pacientes apresentaram resultados acima do limiar.

São vários fatores de risco envolvidos no câncer de próstata, como idade, etnia, histórico familiar, influências ambientais e níveis hormonais. Dentre esses, a idade é o mais expressivo, visto que $70 \%$ dos homens atingidos estão na faixa etária entre 70 e 80 anos, como descrito por Toebe (2020). Diante da análise estatística realizada, observou-se que houve aumento do valor do PSA com o aumento da idade, condizente com a literatura, uma vez que em estudos realizados a grande maioria dos pacientes com valores de PSA alterados tinha mais de 70 anos, como apontado por Souza (2020). Além disso, o risco mais do que duplica quando há um parente de primeiro grau com câncer de próstata como descrito no Oncoguia, no entanto, os valores de PSA encontrados na amostra não foram influenciados por esse fator avaliado.

Cita-se também a raça negra como um fator de risco, descrito no Oncoguia. Na população norte-americana, a incidência e mortalidade do câncer de próstata é 3 e 2,4 vezes maior, respectivamente, em homens negros do que brancos, como apontado por Faria (2020). Perante os dados analisados, não se evidenciaram diferenças no valor do PSA obtido conforme a etnia do paciente. Fatores relacionados à dieta do paciente podem contribuir para uma maior chance de desenvolver câncer de próstata, como ingesta de carne vermelha e laticínios ricos em gordura, como descrito no Oncoguia.

Nos estágios iniciais, a neoplasia prostática é assintomática. Entretanto, em estágios avançados, podem surgir sintomas como dificuldade miccional, disúria, hematúria, hesitação e frequência urinária aumentada, não sendo estes sintomas específicos para a doença. Inclusive, o aumento da frequência urinária é mais comumente causado por hiperplasia prostática benigna, como apontado no Oncoguia. Somado a isso, alguns estudos demonstraram que o rastreamento parece aumentar falsamente a sobrevida, ou seja, não há adiamento do momento de óbito, como descrito por Jansen (2013), e, por consequência, o diagnóstico precoce reduz a qualidade de vida do paciente, com os efeitos colaterais do tratamento e a ansiedade gerada pelo diagnóstico, como visto por Steffen (2018). Muitos pacientes diagnosticados são submetidos a procedimentos com alta morbi-mortalidade, que também 
geram custos financeiros desnecessários, como descrito por Chou (2011). Ademais, outro aspecto preocupante destacado no rastreamento do câncer de próstata, é o sobrediagnóstico, isto é, a detecção de lesões que não seriam identificadas ao longo da vida do paciente, que poderia morrer por outras causas antes de ocorrerem sintomas clínicos da neoplasia, como descrito por Steffen (2018). Em estudos em autópsias, cerca de metade dos homens acima de 80 anos possuíam células malignas na próstata, apesar de não apresentarem nenhum sintoma em vida, como visto por Grossman (2018). Na teoria, o câncer de próstata será incidente em 100\% dos homens que viverem até os 100 anos de idade, portanto, a evolução da doença não afetará a qualidade ou sobrevida do paciente e não será necessariamente a causa do óbito, como descrito por Santos (2020).

Entretanto, é válido ressaltar que existem literaturas que defendem o rastreio do câncer prostático, afirmando que há redução da mortalidade com o rastreio do PSA e que há mais benefícios quanto maior o tempo de seguimento, ou seja, é um processo contínuo, havendo pouco proveito em um rastreio único, como visto por Hugosson (2019). Estima-se evitar duas mortes para cada 1000 homens que são submetidos ao rastreamento, a partir da dosagem de PSA, como descrito por Grossman (2018).

A American Urological Association, em seu guideline para detecção precoce do câncer de próstata, publicado em 2013 e atualizado em 2018, evidencia que quase todos os estudos que avaliaram a triagem baseada em PSA demonstraram benefícios em termos de estágio inferior e grau de câncer no diagnóstico. Além disso, o European Randomized Study of Prostate Cancer avaliou mais de 182 mil homens e, naqueles submetidos à triagem, a mortalidade específica por câncer de próstata foi significativamente menor, como visto pela American Urological Association.

O direcionamento para homens de 55 a 69 anos representa a faixa etária de abordagem com maior evidência de benefício. Não se recomenda rotina de rastreamento em homens entre 40 e 54 anos, com exceção daqueles que apresentam risco elevado de câncer de próstata. Nos indivíduos entre 55-69 anos, deve haver o balanço entre redução da taxa de câncer de próstata metastático e mortes com os potenciais malefícios associados ao rastreio e modalidades de tratamento, como descrito pela American Urological Association.

Cita-se também que, em 2012, a US Preventive Services Task Force realizou uma recomendação contrária ao rastreamento do câncer de próstata através do PSA, independentemente da idade do paciente, como apontado por Moyer (2012), pois inferiu que os malefícios do rastreamento seriam os malefícios gerados pela biópsia necessária para realizar o diagnóstico, como descrito por Wroclawski (2017). Posteriormente a isso, evidenciou-se um aumento na ocorrência de metástases à distância do câncer de próstata em indivíduos com idade a partir de 75 anos, como apontado por Patel (2017). Além de que, atualmente, há forte perspicuidade para indicação de vigilância ativa em pacientes com câncer de próstata de baixo risco, atenuando as complicações do overtreatment, como visto por Wroclawski (2017). A mais recente recomendação da US Task Force indica que a decisão do rastreio deve ser feita de forma individualizada em pacientes com faixa etária entre 55 e 69 anos, juntamente com vigilância ativa, para diagnosticar o câncer de próstata em estágio inicial e, ao mesmo tempo, reduzir sobrediagnóstico e overtreatment como descrito por Patel (2019).

$\mathrm{Na}$ amostra que compõe esse artigo, nenhum paciente foi submetido à biópsia, não havendo nenhum diagnóstico. Podese justificar isso pela faixa etária dos indivíduos, uma vez que a média de idade foi de 51,2 anos e $75 \%$ dos casos ocorrem após os 65 anos e também por ser uma amostra pequena.

\section{Conclusão}

De modo geral, a prevalência dos pacientes que apresentaram PSA > 2,5 ng/dL foi cerca de 3,2\% e nenhum paciente foi submetido à biópsia prostática, não ocorrendo nenhum diagnóstico de câncer de próstata na amostra populacional deste artigo. Diante dos dados apresentados, a individualização no rastreamento do câncer de próstata é mais importante que o rastreamento populacional indiscriminado. Sugere-se a realização de estudos que realizem a biópsia prostática. 


\section{Referências}

Brasil. Resolução 466, de 12 de dezembro de 2012. Aprova diretrizes e normas regulamentadoras de pesquisas envolvendo seres humanos. Brasília: Conselho Nacional de Saúde, 2012

INCA. (2018). Câncer de próstata. INCA - Instituto Nacional de Câncer. https://www.inca.gov.br/tipos-de-cancer/cancer-de-prostata

Castro, H. A. S. D., Iared, W., Shigueoka, D. C., Mourão, J. E. \& Ajzen, S. (2011). Contribuição da densidade do PSA para predizer o câncer da próstata em pacientes com valores de PSA entre 2, 6 e 10, 0 ng/ml. Radiologia Brasileira, 44, 205-9

Chou, R., Croswell, J. M., Dana, T., Bougatsos, C., Blazina, I., Fu, R., Gleitsmann, K., Koenig, H. C., Lam, C., Maltz, A., Rugge, J. B. \& Lin, K. (2011). Screening for Prostate Cancer: A Review of the Evidence for the U.S. Preventive Services Task Force. Annals of Internal Medicine, 155(11), 762. https://doi.org/10.7326/0003-4819-155-11-201112060-00375

De Souza Oliveira, E., da Paz Dias, M., Gomes Filho, E. R., Barbosa, N. B., \& de Morais, A. C. L. N. (2020). Panorama clínico de pacientes submetidos à dosagem de antígeno prostático específico (PSA). Research, Society and Development, 9(9), e423997183-e423997183.

Demuner, B. B., \& Carrijo-Carvalho, L. C. (2021). Avaliação de fatores de risco e antígeno prostático específico no rastreamento de câncer de próstata. Revista de Ciências Médicas e Biológicas, 20(2), 235-239.

Dos Santos Júnior, C. J., \& Tôrres Oliveira, E. C. (2020). [ID 51955] Morbimortalidade por câncer de próstata em homens adultos em um estado do nordeste do brasil: caracterização epidemiológica e análise de tendência temporal. Revista Brasileira de Ciências Da Saúde, 24(4). https://doi.org/10.22478/ufpb.2317$6032.2020 \mathrm{v} 24 \mathrm{n} 4.51955$

Faria, L., Pereira, P., Lustosa, A., Aragão, I., Aragão, F., \& Cunha, M. (2020). Perfil epidemiológico do c ncer de próstata no brasil: retrato de uma década. Revista Uningá, 57(4), 76-84. Recuperado de http://revista.uninga.br/index.php/uninga/article/view/3336

Fontelles, M. J., Simões, M. G., Farias, S. H., \& Fontelles, R. G. S. (2009). Metodologia da pesquisa científica: diretrizes para a elaboração de um protocolo de pesquisa. Revista paraense de medicina, 23(3), 1-8.

Grossman, D. C., Curry, S. J., Owens, D. K., Bibbins-Domingo, K., Caughey, A. B., Davidson, K. W., \& US Preventive Services Task Force. (2018). Screening for prostate cancer: US Preventive Services Task Force recommendation statement. Jama, 319(18), 1901-1913.

Hugosson, J., Roobol, M. J., Månsson, M., Tammela, T. L., Zappa, M., Nelen, V., \& Auvinen, A. (2019). A 16-yr Follow-up of the European Randomized study of Screening for Prostate Cancer. European urology, 76(1), 43-51.

Ilic, D., Neuberger, M. M., Djulbegovic, M., \& Dahm, P. (2013). Screening for prostate cancer. The Cochrane Database of Systematic Reviews, 1, CD004720. https://doi.org/10.1002/14651858.CD004720.pub3

Jansen, R. J., Alexander, B. H., Anderson, K. E., \& Church, T. R. (2013). Quantifying lead-time bias in risk factor studies of cancer through simulation. Annals of epidemiology, 23(11), 735-741.

Modesto, A. A. D. A., Lima, R. L. B. D., D’Angelis, A. C., \& Augusto, D. K. (2017). Um novembro não tão azul: debatendo rastreamento de câncer de próstata e saúde do homem. Interface-Comunicação, Saúde, Educação.

Moyer, V. A. (2012). Screening for Prostate Cancer: U.S. Preventive Services Task Force Recommendation Statement. Annals of Internal Medicine, 157(2), 120. https://doi.org/10.7326/0003-4819-157-2-201207170-00459

Mulhem, E., Fulbright, N., \& Duncan, N. (2015). Prostate Cancer Screening. American Family Physician, 92(8), 683-688. https://pubmed.ncbi.nlm.nih.gov/26554408/

Oncoguia, I. Fatores de Risco para Câncer de Próstata. Instituto Oncoguia. http://www.oncoguia.org.br/conteudo/fatores-de-risco-para-cancer-deprostata/5850/1130/

Oncoguia, I. Sinais e Sintomas do Câncer de Próstata. Instituto Oncoguia. http://www.oncoguia.org.br/conteudo/sinais-e-sintomas-do-cancer-deprostata/1188/289/

Patel, N. A., Sedrakyan, A., Bianco, F., Etzioni, R., Gorin, M. A., Hsu, W. C., \& Hu, J. C. (2019, December). Definitive and sustained increase in prostate cancer metastases in the United States. In Urologic Oncology: Seminars and Original Investigations. 37(12), 988-990. Elsevier.

Prostate Cancer: Early Detection Guideline - American Urological Association. Www.auanet.org. https://www.auanet.org/guidelines/guidelines/prostate-cancerearly-detection-guideline

Sarris, A. B., Candido, F. J. L. F., Pucci Filho, C. R., Staichak, R. L., Torrani, A. C. K., \& Sobreiro, B. P. (2018). Câncer de próstata: uma breve revisão atualizada. Visão Acadêmica, 19(1).

Silva, F. A. da, Santos, F. C. B. dos, Sanches, M. H., Machado, R. L. D., \& Furini, A. A. D. C. (2017). Serum level of prostate specific antigen in users of a clinical laboratory of Novo Horizonte, São Paulo. Revista Brasileira de Análises Clínicas, 49(1). https://doi.org/10.21877/2448-3877.201600526

Sociedade Brasileira de Urologia. Nota oficial 2017 - Rastreamento do Câncer de Próstata. Disponível em: https://sbu-sp.org.br/medicos/noticias/nota-oficial2017-rastreamento-do-cancer-de-prostata/

Steffen, R. E., Trajman, A., Santos, M., \& Caetano, R. (2018). Rastreamento populacional para o câncer de próstata: mais riscos que benefícios. Physis: Revista de Saúde Coletiva, 28, e280209. 
Research, Society and Development, v. 10, n. 17, e100101724465, 2021

(CC BY 4.0) | ISSN 2525-3409 | DOI: http://dx.doi.org/10.33448/rsd-v10i17.24465

Toebe, A. L., Figueró, N. A., Buhring, C. A. Z., \& Mendes, G. A. (2020). Câncer de próstata: revisão da literatura acerca dos diversos aspectos da doença. Anais do Seminário Interinstitucional de Ensino, Pesquisa e Extensão.

Wroclawski, M. L. (2017). New US Preventive Service Task Force recommendations for prostate cancer screening: a needed update, but not enough. Einstein (São Paulo), 15(3), 7-10. https://doi.org/10.1590/s1679-45082017ed4151 